\title{
FRICTION STRESS AND RECOVERY IN CREEP \\ OF NICKEL-BASE SUPERALLOYS
}

\author{
M. McLean \\ Division of Materials Applications \\ National Physical Laboratory \\ Teddington, Middlesex, UK.
}

A new method of chalysing stress decrement creep lesls to determine friction stress $\sigma_{0}$ during high temperature creep has been applied to an experimental nickel-base superalloy and to the $\gamma-\gamma^{\prime}-\mathrm{Cr}_{3} \mathrm{C}_{2}$ in situ composite. $\sigma_{0}$ has been determined as a function of initial stress, temperature and microstmetural variables. The results suggest that oo can be identified with distocation climb around $\gamma^{\prime}$ particies and orowan bowing between carbide fibres.

\section{INTRODUCTION}

The unreasonably high values of stress exponent $\mathrm{n}$ and activetion energy $Q$ that result from expressing the creep data of complex alloys, such as superalloys, by the conventional Bailey-Norton equation (1) can be reduced to levels that are compatible with current concepts of creep (i.e. $n \sim 4, Q \sim$ activation energy for self-diffusion) by expressing the Bailey-Norton equation in terms of an effective $\left(\sigma-\sigma_{0}\right)$, rather than applied stress $\sigma(2,3)$,

$$
\dot{\varepsilon}=A\left(\sigma-\sigma_{0}\right)^{\mathrm{n}} \exp (-Q / R T)
$$

where $\dot{\varepsilon}$ is the minimum creep rate, RT is the thermal energy and $A$ is a constant. Some authors assume the validity of Equation 1 and treat $\sigma$ as an adjustable parameter to get optimum fit of data (49; others attempt to measure $\sigma_{0}$ independently by interpretation of transient creep behaviour $(2,5,6)$. A though creep of engineering alloys is well described by Equation 1 (7) there is no consensus over either the physical significance of $\sigma_{0}$ or the validity of methods proposed to measure it.

Wilshire and co-workers $(2,5,6)$ have pioneered an 
approach to the measurement of $\sigma_{0}$, termed by them a "friction" stress, involving the interpretation of strain transients following successive small reductions in stress. The sum of the periods of zero creep following each stress reduction $\left[\Delta t\right.$ increases rapidly and $\sigma_{0}$ is defined as the stress remaining on the specimen when $[\Delta t \rightarrow \infty$. The simplicity of execution and interpretation of such experiments, where $\Delta t$ is thought to be controlled by the kinetics of recovery of the dislocation substructure, has considerable attractions; however, the practicality of the method has been criticised because of the difficulty of extrapolation from the data to identify the asymptotic valuc of strcss as $\sum \Delta t$ ro $(8,9)$.

In this paper an objective method of measuring $\sigma_{0}$, from stress dip tests, based on concepts of recovery creep, will be described and tcsted with data for an experimental superalloy and an in situ composite. $\sigma_{0}$ will be determined as fuctions of temperature, stress and microstructural variables in these alloys and the results will be compared with theoretical estimates of strengthening in superalloys in order to identify the origins of $\sigma_{0}$.

\section{THE MODEL AND ITS IMPLICATIONS}

The model extends the concepts of recovery controlled creep (10) where at steady state there is a balance between strain hardening and recovery that leads to the establishment of a characteristic dislocation substructure. On making a discontinuous reduction in stress $\Delta \sigma$ the Orowan condition for glide of dislocation segments in the network established under the higher stress is no longer satisfied. Consequently, there is a period of zero creep $\Delta t$ during which the dislocation substructure coarsens until glide of the longest links is possible under the reduced stress. McLean (11) has developed Friedel's model (12) of recovery, taking into account the heterogeneous nature of network growth, to express $\Delta t$ in terms of the initial, residual and friction stresses (i.e. $\sigma_{I}, \sigma_{R}$ and $\sigma_{o}$ resp).

$$
\Delta t=\frac{\mu^{2} b^{2}}{K}\left[\left(\sigma_{R}-\sigma_{0}\right)^{-2}-\left(\sigma_{I}-\sigma_{0}\right)^{-2}\right]
$$

where $\mu=$ shear modulus, $b=$ Burgers vector and $\mathrm{K}=$ kinetic constant that is proportional to the lattice diffusion coefficient.

Equation 2 should remain valid for multiple stress reductions with $\Delta t$ denoting the sum of the delay periods following all preceding stress reductions, provided that 
successive changes in stress are made as soon as forward creep is detected and before substantial changes in the dislocation substructure can occur. The experimental data from the stress decrement creep tests described, for example, by Parker and Wilshire (5), which comprise $\left(\sigma_{I}, \sigma_{R}, \Delta t\right)$, may be fitted by least squares analysis to Equation 3 in order to determine $\sigma_{0}$ and the composite term $\mu^{2} b^{2} / K$.

The typical set of data and the best fit of Equation 2 to it (Figure 1) clearly show close agreement between theory and experiment. Although the curve appears to have flattened out at a value of $\sigma_{\mathrm{R}} \sim 118 \mathrm{MPa}$ within the timescale indicated, it extrapolates very slowly to a true asymptote at $\sigma_{R} \sim 101 \mathrm{MPa}$ which may be identified with $\sigma_{0}$. The rate at which the asymptote is approached is dependent on the magnitude of $\mu^{2} b^{2} / \mathrm{K}$, which has a value of $1.80 \times 10^{7} \mathrm{~N}^{2} \mathrm{smm}^{-4}$ for the $1173 \mathrm{~K}$ data shown in Figure 1. This indicates that previous empirical methods of analysis will always overestimate $\sigma_{0}$. Moreover, the error introduced will be a strong function of temperature and is also likely to depend on $\sigma_{I}$. Consequently, care must be taken in attributing apparent variations in $\sigma_{0}$ estimated by inspection of the experimental data, to functional dependenceson temperature and $\sigma_{I}$ when they may be artefacts of the data analysis.

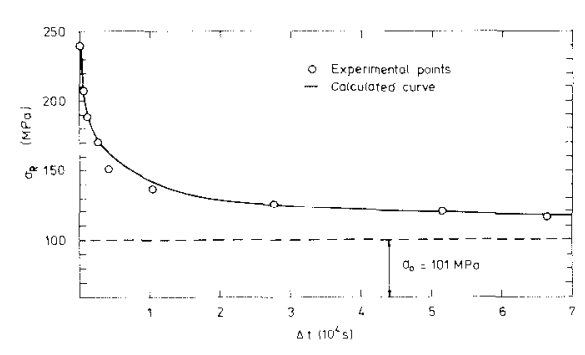

Fig. $1 \quad \sigma_{R}$ vs. $\Delta t$ for $\gamma-\gamma^{\prime}-\mathrm{Cr}_{3} \mathrm{C}_{2}$
The consequences of small changes in $\mu^{2} \mathrm{~b}^{2} / \mathrm{K}$, $\sigma_{I}$ and $\sigma_{0}$ to Equation 2, for the parameters of Figure 1, are examined in Figure 2. It is of interest to consider how changes in these variables would influence the apparent $\sigma_{0}$ determined by previous empirical methods in the time scale of typical stress dip creep tcsts. Clearly substantial changes to the curves can

result from variations of any of the three parameters and interpretation in terms of only a change in $\sigma_{0}$ could be erroneous.

\section{RESUITS}

The experimental data were obtained using the procedures given by Parker and Wilshire (5) and described in detail elsewhere (11). Measurements were made on (i) the $\gamma^{-} \gamma^{\prime}-\mathrm{Cr}_{3} \mathrm{C}_{2}$ 
in situ composite which consists of $\gamma$-nickel reinforced by $\gamma^{\prime}-\mathrm{Ni}_{3} \mathrm{Al}$ particles and $\mathrm{Cr}_{3} \mathrm{C}_{2}$ fibres, and (ii) an experimental $\gamma-\gamma^{\prime}$ superalloy obtained by eliminating the carbide from $\gamma^{-} \gamma^{\prime}-\mathrm{Cr}_{3} \mathrm{C}_{2}$. The compositions of both alloys, which were
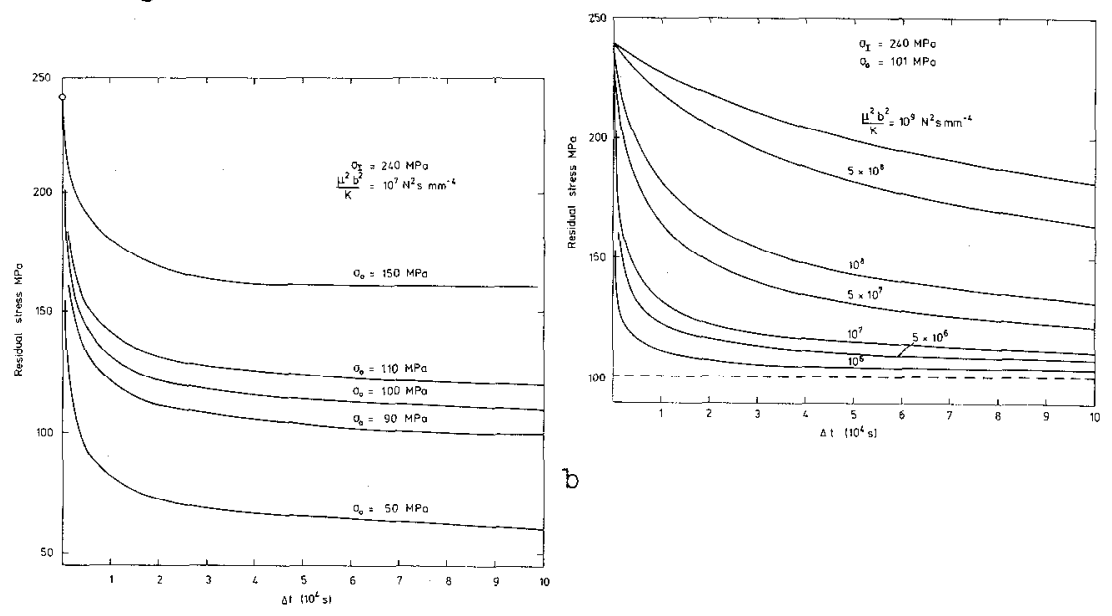

b

a

Fig.2 Modification of transient creep behaviour following a stress reduction with changing (a) $\sigma_{O},(\mathrm{~b}) \mu^{2} \mathrm{~b}^{2} / \mathrm{K}$ and (c) $\sigma_{I}$. The figures arc based on parameters for $\gamma-\gamma^{\prime}-\mathrm{Cr}_{3} \mathrm{C}_{2}$ at $1173 \mathrm{~K}$.

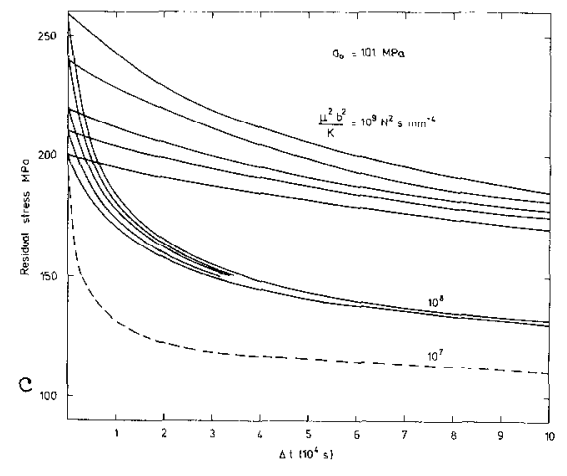

directionally solidified as described by Bullock et al (13), are given in Table 1.

Table 1 Alloy compositions (weight per cent)

\begin{tabular}{lcccc} 
& $\mathrm{Ni}$ & $\mathrm{Al}$ & $\mathrm{Cr}$ & $\mathrm{C}$ \\
\cline { 2 - 5 }$\gamma^{-\gamma^{\prime} \text { superalloy }}$ & 87.54 & 7.65 & 4.28 & 0.52 \\
\hline$\gamma-\gamma^{\prime}-\mathrm{Cr}_{3} \mathrm{C}_{2}$ & 79.00 & 6.90 & 12.34 & 1.76
\end{tabular}

The transient creep behaviour generally consisted of a period of zero creep followed by slowly increasing forward 
creep as reported by previous authors $(5,6)$ and required by the model. The values of $\sigma_{0}$ determined for both $\gamma-\gamma^{\prime}-\mathrm{Cr}_{3} \mathrm{C}_{2}$ and the experimental $\gamma-\gamma^{\prime}$ alloys, using the new method of data analysis, are displayed as functions of $\sigma_{I}$ and the temperature in Figures $3 \mathrm{a}$ and $\mathrm{b}$. These values of $\sigma_{0}$ appear to be invariant over the limited range of initial stresses for both alloys and of temperature for the $\gamma^{\prime} \gamma^{\prime}$ superalloy whereas simple inspection of the dat.a. would suggest, significant variations. However, there is a systematic trend of reducing $\sigma_{0}$ with increasing temperature for $\gamma-\gamma^{\prime}-\mathrm{Cr}_{3} \mathrm{C}_{2}$. The microstructural dimensions of the $\gamma-\gamma^{\prime}-\mathrm{Cr}_{3} \mathrm{C}_{2}$ have been varied by changing the principal solidification parameters (i.e. temperature gradient $G$ and solidification rate $R$ ) and Fig. 3c shows that $\sigma_{0}$ decreases with increasing cooling rate GR that leads to finer microstructures. Values of the flow stresses at slow constant strain rates characteristic of creep, previously suggested $(2,15)$ to be equivalent to $\sigma_{0}$, are also included in Figure 2 .

The kinetic constants $\mu^{2} b^{2} / K$ for both alloys considered in this study are displayed as a function of temperature as Arrhenius-type plots (Fig. 4); values for other nickel-base superalloys calculated from published data are also included. The points fall on a band that indicates an activation energy of $265 \mathrm{~kJ} \mathrm{~mol}^{-1}$ which is close to that for self-diffusion in nickel (14).

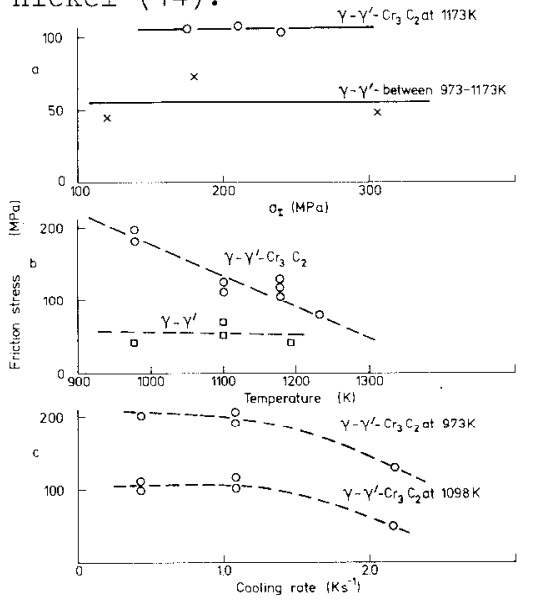

Fig. $3 \sigma_{0}$ as functions of

(a) $\sigma_{\text {I }}$
(c) comperature and

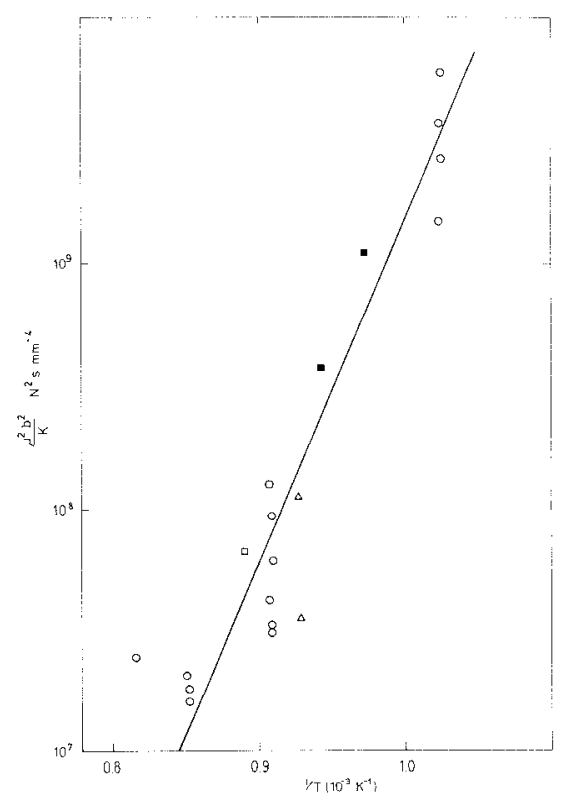

Fig. $4 \log \mu^{2} b^{2} / K$ vs. $1 / T$ 


\section{DISCUSSION}

The observation of delay periods, the accurate description of transient creep by Equation 2 and the apparent universality of Fig. 4 for Ni-base superalloys (indicating $Q$ for nickel self-diffusion) cumulatively support the concept that creep is recovery controlled for the lest conditions considerea. This requires lhal dislocation motion during recovery, motivated by dislocation line tension, be much slower than during the propagation of suitable links by the applied stress. Consequently the mechanism for the propagation stage, whether diffusive or glide, should have little influence on the creep behaviour and care must be taken in interpreting microstructural evidence of a particular deformation mechanism (e.g. anti phase boundaries due to particle shearing) as evidence of its dominance. The propagation stage may be slower than recovery for other test conditions when the model proposed above will be inappropriate. This will occur when the equivalent stress due to the dislocation network $\sigma_{E}$ exceeds the applied stress.

The procedure of monitoring the delay periods following successive stress reductions essentially characterises the recovery process and provides a measure of the rate of relaxation of the dislocation substructure in approaching a balance between the applied stress and the inherent resistance of the alloy to deformation. The practical lower limit to the detection of creep will determine how close the final stress approaches $\sigma_{0}$ and, therefore, the experimental accuracy of the method when extrapolation procedures are not used. The applied stress $\sigma^{\prime}$ leading to the minimum detectable creep rate $\dot{\varepsilon}^{\prime}$ may be expressed in terms of Equation 1,

$$
\sigma^{\prime}-\sigma_{0}=\left(\frac{\dot{\varepsilon}^{\prime}}{A}\right)^{\frac{1}{4}} \exp \left(\frac{Q}{4 R T}\right)
$$

Taking $\quad \dot{\varepsilon}^{\prime}=10^{-10} \mathrm{~s}^{-1}$ as characteristic of the resolution of modern creep testing and values of $Q=265 \mathrm{kJmol}-1$ and

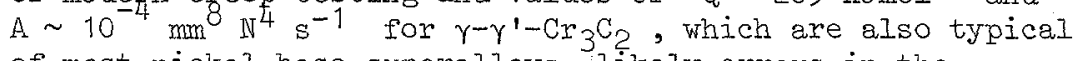
of most nickel-base superalloys, likely errors in the measurement of $\sigma_{0}$ by previous empirical methods have been calculated and are listed in Table 2 as a function of temperature. Clearly very substantial errors are introduced unless reliable methods of extrapolation beyond the data are used. The procedure described in this paper appears to go a long way to reducing these errors. 
Table 2 Difference between $\sigma_{0}$ and final stress $\sigma^{\prime}$ during stress dip tests for $\gamma-\gamma^{\prime}-\mathrm{Cr}_{3} \mathrm{C}_{2}$

\begin{tabular}{lllll} 
Temperature $K$ & 973 & 1073 & 1173 & 1273 \\
\hline$\sigma^{\prime}-\sigma_{0} \mathrm{MPa}$ & 90 & 42.9 & 23.2 & 13.8
\end{tabular}

In the superalloy, the lack of sensitivity of $\sigma_{0}$ to applied stress and temperature taken with the equivalence of $\sigma_{0}$ and flow stress strongly suggests that friction stress in such materials derives principally from the intrinsic structure of the alloy (e.g. precipitate particles, solute atoms) rather than from the dislocation substructure. The $\gamma^{\prime}$ particles are likely to constitute the major barriers to deformation and $\sigma_{0}$ may be related to the strength of these obstacles. Various mechanisms for by-passing of the precipitate particles by dislocations have been proposed (17) (e.g. dislocation climb, particle shearing, bowing between particles) and that leading to the highest deformation rate should dominate. The threshold stresses associated with various particle-by-passing modes have been calculated by Mclean (18) using parameters relevant to the alloys under consideration. Comparison of $\sigma_{0}$ with the calculated flow stresses (Table 3) suggests that the friction stress in the superalloy arises from dislocation climb around $\gamma^{\prime}$ particles, while in the $\gamma^{-} \gamma^{\prime}-\mathrm{Cr}_{3} \mathrm{C}_{2}$ composite the resistance to flow has components due to climb around $\gamma^{\prime}$ particles and to bowing between carbide fibres. Both models require that $\sigma_{0}$ be weakly dependent on temperature, principally through $\mu$. The present results for the $\gamma^{-\gamma^{\prime}}$ superalloy confirm this expectation, whereas previous reports of a strong dependence of $\sigma_{0}$ on tcmpcraturc may be a consequence of errors in the former methods of data analysis. The strong variation in $\sigma_{0}$ with temperature for the $\gamma^{-} \gamma^{\prime}-\mathrm{Cr}_{3} \mathrm{C}_{2}$ is a result of microstructural changes, involving the transfer of $\gamma^{\prime}$ from the matrix to the carbide surfaces, that predominate at high temperature. Consequently, the lower $\sigma_{o}$ at high temperatures is a reflection of a true weakening of the matrix.

The work presented here has two distinct implications to engineering design. Firstly, it supports the validity of Equation 1 as a relevant constitutive equation describing creep deformation and describes an objective method of measuring $\sigma_{0}$ : This may contribute towards an improved basis for the prediction of service performance. Secondly, although Equation 2 was developed in order to interpret stress dip ereep tests, it should be equally relevant in describing transient deformation during load and temperature excursions 
in service. Consequently, Equation 2 may be regarded as a second constitutive equation that partially accounts for deformation during low cycle stress and thermal fatigue.

\section{CONCLUSIONS}

1. The results of stress dip creep tests on a superalloy and in situ composite are well described by Equation 2 which has been derived using a model of recovery controlled creep, and which provides an objective method of measuring friction stress $\sigma_{0}$.

2. In the superalloy $\sigma_{0}$ is relatively insensitive to changes in initial stress and temperature and appears to correlate with the flow stress for dislocation climb around $\gamma^{\prime}$ particles.

3. In the composite $\sigma_{0}$ varies with temperature and microstructural scale due to phase instabilities, and both dislocation climb around $\gamma^{\prime}$ particles and bowing between carbide fibres contribute to $\sigma_{0}$.

4. The kinetics of recovery of both alloys, and available data for other nickel base superalloys, fit on a single Arrhenius-type plot that indicates an activation energy of $265 \mathrm{~kJ} \mathrm{~mol}^{-1}$.

\section{ACKNOWLFDGFMENTS}

The work reported above was partially supported by the Ministry of Defence (Procurement Executive).

Table 3 Theoretical flow stresses for various particle by-pass mechanisms and measured friction stresses at $1098 \mathrm{~K}$.

\begin{tabular}{lc||cc}
\hline \multicolumn{1}{c}{ Mechanism } & $\begin{array}{c}\text { Theoretical Flow } \\
\text { Stress }\end{array}$ & $\begin{array}{c}\text { Measured } \\
\text { Fraction Stress }\end{array}$ & $\begin{array}{c}\text { Alloy } \\
\mathrm{MPa}\end{array}$ \\
\hline $\begin{array}{l}\text { Dislocation climb } \\
\text { around } \gamma^{\prime}\end{array}$ & 40.7 & 50 & $\gamma-\gamma^{\prime}$ \\
$\begin{array}{l}\text { Particle cutting } \\
\left(\gamma^{\prime}\right)\end{array}$ & 186 & & \\
$\begin{array}{l}\text { Orowan bowing } \\
\begin{array}{l}\text { (a) between } \gamma^{\prime} \\
\text { (b) between } \\
\text { carbides }\end{array}\end{array}$ & 259 & 125 & $\gamma^{-} \gamma^{\prime}-\mathrm{Cr}_{3} \mathrm{C}_{2}$ \\
\hline
\end{tabular}




\section{REFERENCES}

$1 \quad$ O.D. Sherby and P.M. Burke, Progress in Materials Science 13, 325 (1968)

2 P.L. Threadgill and B. Wilshire, Proc. of Iron and Steel Inst. Conf. on "Creep Strength in Steels", Sheffield, 1972 .

3 C.N. Ahlquist and W.D. Nix, Acta Met. 19, 373 (1971).

4 S. Purushothaman and J.K. Tien, Acta Met. 26, 519 (1978).

5 J.D. Parker and B. Wilshire, Metal Sci. 9, 248 (1975).

6 K.R. Wililiams and B. Wilshire, Metal Sci. 1, 176 (1973).

7 W.J. Evans and G.F. Harrison, Metal Sci. 10, 307 (1976).

8 B. Burton, Metal Sci. 9, 297 (1975).

9 J.C. Gibeling and W.D. Nix, Metal Sci. 11, 453 (1977).

10 D. Mclean, Reports of Progress in Physics 29, 1 (1966).

11 M. McLean, Proc. Roy. Soc. (in press).

12 J.Friedel, "Dislocations", Pergamon Press, Oxford $(1964)$.

13 E. Bullock, P.IN. Quested and M. Metean, Proc. of Conf. on "In situ Composites III", Ginn \& Co., Lexington, Mass, 1979.

14 R.E. Hoffman, F.W. Pikus and R.A. Ward, Trans AIME 206, $433(1956)$.

15 T.B. Gibbons, Seripta Met. 12, 749 (1978).

16 T.B. Gibbons and M. Henderson-Brown, Seripta Met 9, 15 (1975).

17 N. Stolloff, "The Superalloys", McGraw-Hill (1972).

18 M. Mclean to be published. 